\title{
Resetting single nanoparticle structural phase with nanosecond pulses
}

\author{
B. F. Soares, ${ }^{1}$ K. F. MacDonald,${ }^{1}$ and N. I. Zheludev ${ }^{1, *}$ \\ ${ }^{1}$ Optoelectronics Research Centre, University of Southampton, SO17 1BJ, United Kingdom
}

(Dated: March 26, 2007)

\begin{abstract}
We demonstrate that a transition from a high-temperature phase to a low-temperature phase in a gallium nanoparticle can be stimulated by nanosecond optical pulses, and explain how this phenomenon may be used in a single-nanoparticle all-optical memory.
\end{abstract}


There is increasing interest in the potential use of phase-change recording materials, [1-3] such as those that form the basis of current DVD/DVDR technology, to address the growing challenges of size and power consumption in electronic memories.[4] In such materials, data recording is achieved by switching between structural forms with differing optical and/or electrical properties using laser or current pulses to attain a temperature in the switched region often as high as $600 \mathrm{~K}$. It has recently been demonstrated that single gallium nanoparticles can act as rewritable optically-addressed memory elements offering storage densities comparable to those expected in next-generation hard disk drives employing perpendicular recording technology, and switching energies an order of magnitude smaller than those employed in current DVD or hard disk technologies. [5, 6] This memory functionality relies on optical bistability resulting from the large overcooling displayed by the gallium nanoparticle system. Different phases of gallium correspond to different logic states, and laser pulses are used to switch the particle from lower to higher energy states. However, in this system the only way to initiate the reverse transformation, i.e. to return to a lower energy state, is to substantially cool the particle (see Fig. 1).

We now report that the overcooling (up to $\sim 95 \mathrm{~K}$ ) observed in a single gallium nanoparticle probed with low-power cw laser light,[7] can be reduced to $\sim 5 \mathrm{~K}$ when the particle is excited with higher intensity, low energy nanosecond laser pulses. This induced narrowing of the hysteresis (see Fig. 1) has important consequences in terms of potential practical applications, particularly where the nanoparticles are employed as memory elements, as it may provide a means of switching in both directions between memory states using only laser light.

A single gallium nanoparticle was grown on the $100 \mathrm{~nm}$ aperture at the tip of a tapered, gold-coated silica optical fiber (see inset to Fig. 2) by attaching the fiber to a liquid nitrogen cryo-stage at $80 \mathrm{~K}$ inside a vacuum chamber evacuated to $10^{-6} \mathrm{mbar}$ and exposing it to a gallium atomic beam with a mass flux of $0.3 \mathrm{~nm} / \mathrm{min}$ for $60 \mathrm{~min}$. In our first experiment (see Fig. 2),[7] a $1310 \mathrm{~nm} \mathrm{cw}$ diode laser (giving $\sim 20 \mathrm{nW}$ at the nano-aperture) was used to probe the reflectivity of the particle, while a $1550 \mathrm{~nm}$ laser $(\sim 30 \mathrm{nW})$ modulated at $f=2.3$ $\mathrm{kHz}$ acted as a pump source. Structural transformations were observed by monitoring pumpinduced changes in the magnitude of the reflected probe signal, as a function of temperature between 80 and $300 \mathrm{~K}$, using a using a lock-in detector referenced to the pump modulation frequency $f$ (a wavelength-division multiplexer (WDM) and band-pass filter prevented the 
detection of reflected pump light).

With increasing temperature, a sequence of narrow positive and negative peaks is seen in the induced-reflectivity change signal between 200 and $250 \mathrm{~K}$ (Fig. 3b), each of which is associated with a structural (phase and/or shape) change in the particle.[7] The signal observed on cooling is rather different (Fig. 3c), comprising a series of relatively small, stepchanges in magnitude at temperatures different from those of the increasing-temperature peaks. If we reasonably assume that the first and last peaks and steps (i.e. $I$ and $A, V$ and $C$ ) are associated with transitions into and out of the highest and lowest temperature phases, we conclude that the particle is subject to overcooling of 45 - $90 \mathrm{~K}$.

For the purposes of the present study, the modulated pump laser was replaced with one operating at the same wavelength but producing 3 ns pulses at $30 \mathrm{kHz}$ with a peak power of $\sim 0.1 \mathrm{~mW}$ at the nano-aperture (average power of the same order as the modulated cw laser). Under these excitation conditions, the particle's response changes dramatically (Fig. 3a). With increasing temperature, peaks $I$ and $V$ are still clearly visible (labelled $I^{\prime}$ and $V^{\prime}$ ) but the intermediate peaks are replaced by smoother, broader features. Most significantly, under the pulsed excitation regime the decreasing temperature response pattern is very similar to the increasing temperature pattern - it shows the same major peaks with overcooling of only $\sim 2 \mathrm{~K}$ for peak $I^{\prime}$ and $\sim 5 \mathrm{~K}$ for peak $V^{\prime}$. The offset of $\sim 65 \mathrm{~K}$ between measured peak positions in Fig.'s 3a and b is the result of laser-induced heating under the short-pulse excitation regime. (Calculations confirm that under such conditions the energy absorbed by a particle cannot dissipate during the pulse and therefore rapidly increases its temperature.)

Substantial changes in the optical properties of a nanoparticle can occur when it undergoes a transition between phases with differing dielectric coefficients[8] (as possessed by gallium's various phases $[9,10])$ or or between different geometrical forms. One may thus detect such transitions in nanoparticles by monitoring their optical properties as described above. Peaks in the pump-induced reflectivity change signal are characteristic of such transitions $[7,11]$ and are related to the nature of the phase transition process in nanoparticles. In bulk solids, phase transitions are characterized by a discontinuous (irreversible) rearrangement of the crystalline lattice at a specific temperature. However, in nanoparticles transitions from lower to higher energy states proceed through a surface-driven dynamic coexistence of forms across a certain temperature range.[12-17] Within the transitional range particles comprise a core in the lower energy phase covered by a 'shell' of the higher energy 
phase, the thickness of which increases with temperature and/or incident light intensity.[18] If the two phases have different dielectric properties, the continuous structural transformation is accompanied by a continuous change in the particle's optical properties. While the phases coexist the transition is reversible, i.e. if the temperature or level of optical excitation is reduced before the core is completely consumed by the new phase, the surface layer will retreat and the particle's optical properties will recover accordingly. Thus, in the present case, modulated optical excitations induce modulations of the particle's optical properties. When the core of a particle is fully converted to the new phase, it becomes stable against a return to the old phase because that would require the creation of a nucleation center. At this point any applied optical excitation abruptly ceases to induce any significant change in the particle's optical properties, until the temperature approaches the next phase transition point.

The need for a nucleation center to initiate the reverse (high- to low-energy phase) transition means that a particle is usually subject to overcooling before undergoing an abrupt transformation to the lower energy state, creating a hysteresis in it's properties as illustrated by Fig.'s 3b and c. While the particle remains in the higher energy state the pump-induced reflectivity change signal is small, and it remains so after the transition because the transition occurs several tens of degrees below the increasing-temperature signal peak. However, when short (ns) pulse excitation is employed, overcooling is all but eliminated and the signal structures in the two directions of temperature are almost identical (Fig. 3a). This near-total disappearance of overcooling may result from the fact that a short, intense pump pulse can help a slightly overcooled particle to transform from a high temperature phase to a lower temperature phase by providing the energy required for it to overcome the potential barrier between the phases. A similar effect is observed in the 'explosive crystallization' of thin metal films, where a localized energy input stimulates an abrupt transition from an overcooled liquid state to the solid state.[19]

The ability to greatly reduce overcooling in a nanoparticle using short, intense optical excitation pulses may be of exceptional importance for future applications. For example, the existence of overcooling leads to a form of optical bistability that enables gallium nanoparticles to act as memory elements, wherein different structural phases represent different logical memory states (see Fig 1).[5, 6] In this earlier work it was reported that single microsecond optical pulses could readily 'write' information to the nanoparticle memory, i.e. switch it 
from a low energy, low logic, state to a higher one, at at fixed temperature $T_{\text {set }}$ within the hysteresis loop (between $T_{0}$ and $T_{1}$ ). 'Reading' the memory state was also achieved optically by probing the particles' reflective properties, however the only way to 'erase' information was to cool the particles to below $T_{0}$. The present study demonstrates that if $T_{\text {set }}$ lies above $T_{0}$ but below $T_{0}^{\prime}$ (the low temperature limit of the narrowed hysteresis loop) then a short, high-intensity, low-energy light pulse might be used to erase the memory without changing its temperature.

To summarize, an investigation of phase transformations induced by nanosecond laser pulses in a single gallium nanoparticle has revealed that the overcooling usually displayed by the metal is greatly reduced (from $\sim 95 \mathrm{~K}$ to $\sim 5 \mathrm{~K}$ ) under such an excitation regime. The finding that phase switching might be optically induced in both increasing and decreasing energy directions in such nanostructures removes a potential barrier to their future application as key logical and bistable memory elements for nanophotonic devices operating at extremely low power levels.

The authors acknowledge the support of the Engineering and Physical Sciences Research Council (UK), the EC-funded STRP-016881 project 'SPANS', and the Foundation for Science and Technology (Portugal). 
* niz@orc.soton.ac.uk; www.nanophotonics.org.uk

[1] M. H. R. Lankhorst, B. W. S. M. M. Ketelaars, and R. A. M. Wolters, Nat. Mat. 4, 347 (2005).

[2] G. A. Gibson, A. Chaiken, K. Nauka, C. C. Yang, R. Davidson, A. Holden, R. Bicknell, B. S. Yeh, J. Chen, H. Liao, S. Subramanian, D. Schut, J. Jasinski, Z. Liliental-Weber, Appl. Phys. Lett. 86, 051902 (2005).

[3] H. F. Hamann, M. O’Boyle, Y. C. Martin, M. Rooks, and H. K. Wickramasinghe, Nat. Mat. 5, 383 (2006).

[4] R. Waser, ed., Nanoelectronics and Information Technology, 2nd ed. (Wiley-VCH, 2005).

[5] B. F. Soares, M. V. Bashevoy, F. Jonsson, K. F. MacDonald, and N. I. Zheludev, Opt. Exp. 14, $10652(2006)$.

[6] B. F. Soares, F. Jonsson, and N. I. Zheludev, Phys. Rev. Lett. 98 (2007, in press).

[7] B. F. Soares, K. F. MacDonald, V. A. Fedotov, and N. I. Zheludev, Nano Lett. 5, 2104 (2005).

[8] V. A. Fedotov, V. I. Emelyanov, K. F. MacDonald, and N. I. Zheludev, J. Opt. A: Pure Appl. Phys. 6, 155 (2004).

[9] M. Bernasconi, G. L. Chiarotti, and E. Tosatti, Phys. Rev. B 52, 9988 (1995).

[10] N. R. Comins, Philos. Mag. 25, 817 (1972).

[11] K. F. MacDonald, V. A. Fedotov, S. Pochon, G. C. Stevens, F. V. Kushmartsev, V. I. Emel'yanov, and N. I. Zheludev, Europhys. Lett. 67, 614 (2004).

[12] R. Garrigos, P. Cheyssac, and R. Kofman, Z. Phys. D 12, 497 (1989).

[13] K. F. Peters, Y.-W. Chung, and J. B. Cohen, Appl. Phys. Lett. 71, 2391 (1997).

[14] G. B. Parravicini, A. Stella, P. Tognini, P. G. Merli, A. Migliori, P. Cheyssac, and R. Kofman, Appl. Phys. Lett. 82, 1461 (2003).

[15] R. S. Berry and B. M. Smirnov, J. Chem. Phys. 113, 728 (2000).

[16] P. Buffat and J.-P. Borel, Phys. Rev. A 13, 2287 (1976).

[17] A. S. Shirinyan and M. Wautelet, Nanotechnology 15, 1720 (2004).

[18] M. Wautelet, J. Phys.: Condens. Matter 16, L163 (2004).

[19] V. M. Kuz'menko, V. I. Mel'nikov, and V. A. Rakhubovskii, Sov. Phys. JETP 59, 612 (1984). 


\section{Figure captions:}

FIG 1. Reflectivity hysteresis and memory functionality (logic states '0' and '1') in a gallium nanoparticle. Solid line: schematic of the reflectivity hysteresis resulting from the extensive overcooling of the high-energy phase usually required before a transition to the low-energy phase occurs. Dashed line: hysteresis narrowing under ns pulsed optical excitation.

FIG 2. Experimental arrangement for reflective measurements to study excitationinduced structural changes in a single gallium nanoparticle located on the aperture at the tip of a tapered, gold-coated silica optical fiber. The inset scanning electron microscope images show such a fiber tip before and after gallium particle formation.

FIG 3. Magnitude of pump-induced change in the reflectivity of a single gallium nanoparticle (a) subject to ns pulsed pump excitation, as a function of increasing and decreasing temperature; (b) subject to modulated cw pump excitation with a peak power of $\sim 30 \mathrm{nW}$, as a function of increasing temperature; (c) subject to modulated cw pump excitation, as a function of decreasing temperature. $\mathrm{cw}$ probe power at aperture $\sim 20 \mathrm{nW}$ in all cases. 
Erasing ' 1 ' to ' 0 '

by cooling
Erasing ' 1 ' to ' 0 ' with ns pulses
High energy phase (' 1 ')

Low energy

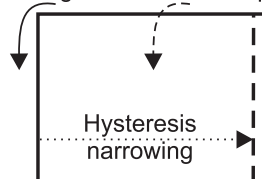
phase (' 0 ') 


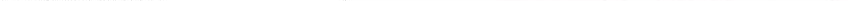




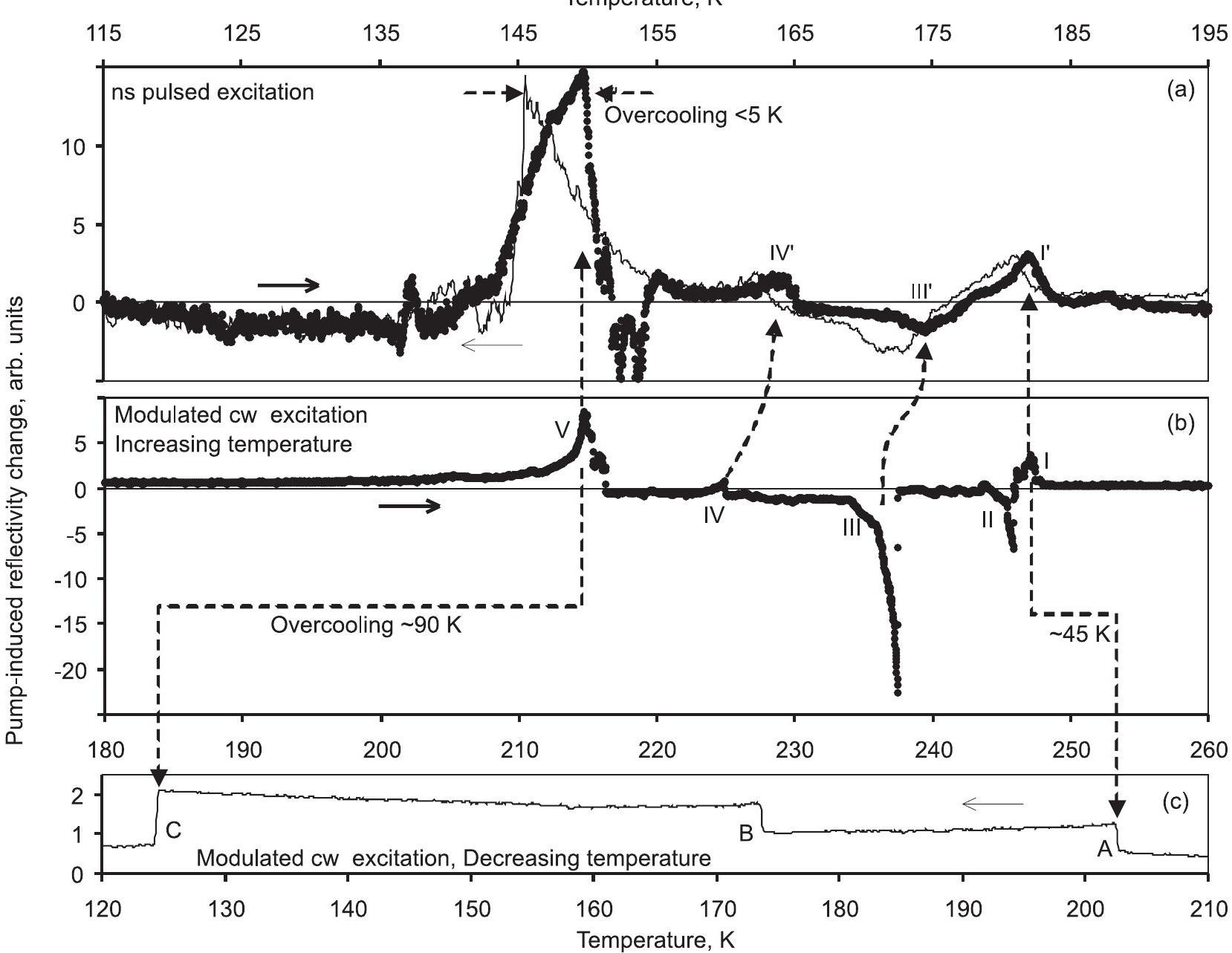

